\title{
Science with the James Webb Space Telescope
}

\author{
Jonathan P. Gardner \\ Exploration of the Universe Division, Observational Cosmology Laboratory, Code 665, \\ Goddard Space Flight Center, Greenbelt MD 20771, USA \\ email: jonathan.p.gardner@nasa.gov
}

\begin{abstract}
The scientific capabilities of the James Webb Space Telescope (JWST) fall into four themes. The End of the Dark Ages: First Light and Reionization theme seeks to identify the first luminous sources to form and to determine the ionization history of the universe. The Assembly of Galaxies theme seeks to determine how galaxies and the dark matter, gas, stars, metals, morphological structures, and active nuclei within them evolved from the epoch of reionization to the present. The Birth of Stars and Protoplanetary Systems theme seeks to unravel the birth and early evolution of stars, from infall onto dust-enshrouded protostars, to the genesis of planetary systems. The Planetary Systems and the Origins of Life theme seeks to determine the physical and chemical properties of planetary systems around nearby stars and of our own, and investigate the potential for life in those systems. To enable these four science themes, JWST will be a large $(6.5 \mathrm{~m})$ cold $(50 \mathrm{~K})$ telescope launched to the second Earth-Sun Lagrange point early in the next decade. It is the successor to the Hubble Space Telescope, and is a partnership of NASA, ESA and CSA. JWST will have four instruments: The Near-Infrared Camera, the Near-Infrared multi-object Spectrograph, and the Tunable Filter Imager will cover the wavelength range 0.6 to 5 microns, while the Mid-Infrared Instrument will do both imaging and spectroscopy from 5 to 27 microns. The scientific investigations described here define the measurement capabilities of the telescope, but they do not imply that those particular observations will be made. JWST is a facility-class mission, so most of the observing time will be allocated to investigators from the international astronomical community through competitively-selected proposals.
\end{abstract}

Keywords. Galaxies: formation, Stars: formation, planetary systems, Space vehicles: instruments.

\section{The end of the Dark Ages: first light and reionization}

JWST seeks to identify the first luminous sources to form and to determine the ionization history of the early universe. The emergence of the first sources of light in the universe marks the end of the "Dark Ages" in cosmic history, a period characterized by the absence of discrete sources of light (Rees 1997). Some time after the appearance of the first sources of light, hydrogen in the intergalactic medium was reionized. Results from the Wilkinson Microwave Anisotropy Probe (Kogut et al. 2003) combined with data on quasars at $z \sim 6$ from the Sloan Digital Sky Survey (Fan et al. 2002) show that this reionization had a complex history (Cen 2003). Although there are indications that galaxies produced the majority of the ultraviolet radiation which caused the reionization, the contribution of quasars could be significant.

\subsection{What are the first galaxies?}

To identify a sample of high redshift galaxies, JWST will make an ultra-deep imaging survey using several broad-band filters. The Lyman break technique will identify objects at increasing redshifts up to $z=30$ or higher. For dwarf galaxies with $10^{6} \mathrm{M}_{\odot}$ of 
zero-metallicity massive stars at $15<z<30$, the expected AB magnitude at emitted wavelengths just longward of Lyman $\alpha$ is $\sim 31$ mag. This ultra-deep survey will allow the derivation of galaxy counts as a function of redshift, seeing a drop at the formation of the first sources. The intensity of the non-ionizing continuum can be calibrated to yield the star formation rate as a function of redshift. The deep broad-band imaging in the near infrared will enable follow-up low-resolution spectroscopy and mid-infrared imaging of promising sources. The imaging could be timed to search for supernovae.

\subsection{When and how did reionization occur?}

JWST will obtain high signal-to-noise, $\mathrm{R}=1000$, near-infrared spectra of high-redshift QSOs or galaxies in order to determine the presence of a Gunn-Peterson trough or of a Lyman $\alpha$ damping wing. The targets will be the brightest known high redshifts objects. A damping wing should be present for a few million years, before the ionizing radiation is sufficient to create a large Strömgren sphere around each ionizing source. If there are indeed two distinct reionization epochs, the (possibly partial) recombination following the first reionization may be detectable in continuum spectra of high redshift objects as an absorption signature in the region shortward of Lyman $\alpha$.

In order to measure the ionizing continuum of a class of sources, we need to measure their hydrogen and helium Balmer lines. Comparison between these lines gives an estimate for the steepness, or hardness of the ionizing continuum, providing a measurement of the rate of production of ionizing photons for any given class of sources under the assumption that the escape fraction is known. The escape fraction can be measured from deep imaging observations, or estimated from the line equivalent widths. This program requires near-infrared spectroscopy of very faint objects. The expected observed surface brightness of the sources responsible for reionization ranges between $\mathrm{AB}=27$ and $29 \mathrm{mag}$ $\operatorname{arcmin}^{-2}$, depending on the escape fraction, and is counted as the typical ionizing flux per unit area over which they are detected. Identification of the nature of ionizing sources requires a combination of diagnostics: line shapes, line widths, line ratios, and the shape of the continuum. We expect the intrinsic line shapes and widths of AGN-powered sources to be broader than those of sources ionized by stellar radiation.

\section{The assembly of galaxies}

JWST seeks to determine how galaxies and the dark matter, gas, stars, metals, morphological structures, and active nuclei within them evolved from the epoch of reionization to the present day. Theory and observation show that galaxies are assembled through a process of the hierarchical merging of dark matter concentrations, accompanied by chemical evolution of the gas and dust, and resulting in the Hubble Sequence of galaxies. However, we do not really know how galaxies are formed, what controls their shapes, and what makes them form stars. We do not know how the chemical elements are generated and redistributed through the galaxies, and whether the central black holes exert great influence over the galaxies. We do not know the global effects of violent events as small and large parts join together in collisions.

\subsection{How did the Hubble Sequence and heavy elements form?}

To trace the formation of the Hubble Sequence of galaxies, JWST will determine the morphologies, stellar populations, and star-formation rates in a very large sample of galaxies observed in deep-wide imaging and spectroscopic surveys, as a function of their mass, environment, and cosmic epoch. JWST will also determine when the long-lived stars in 
a typical galaxy were formed, whether in situ or in smaller galaxies that subsequently merged together to form a large galaxy. Direct characterization of the merging rate of galaxies will provide another angle on this question.

The average metallicity of the universe and of the objects in it as a function of epoch provides a fundamental metric reflecting the development of structure and complexity on galactic scales. The metallicities of star-forming gas, especially of the $[\mathrm{O} / \mathrm{H}]$ abundance, can be measured using diagnostics such as the $\mathrm{R}_{23}$ index, which is based on strong emission lines. Such measurements require $\mathrm{R} \sim 1000$ to separate $\mathrm{H} \alpha$ and [NII]. JWST will measure rest-frame optical emission lines from compact low-extinction galaxies at $z \sim 5$ forming stars at a rate comparable to that of the Milky Way today. In order to assemble sufficient samples for statistical determinations, JWST will be able to make these measurements with high multiplexing gain.

\subsection{What physical processes determine galaxy properties?}

Despite the variety of galaxy properties observed today, galaxies obey a number of remarkably tight scaling relations between basic properties of luminosity, size, kinematics and metal enrichment. These include the Tully-Fisher relation for disk galaxies and the "fundamental plane", and projections thereof, for spheroids. Most recently, a surprising relationship between the mass of the central black hole and the properties of the surrounding spheroid (e.g., the velocity dispersion) has been established (e.g., Ferrarese \& Merritt 2000). JWST will investigate these processes through deep imaging for structural parameters plus high-resolution spectroscopy for kinematical data. The dark matter will be revealed through gravitational weak-lensing surveys reaching $z \sim 2.5$.

The optical identification of high-redshift Ultra-Luminous Infra-Red Galaxies (ULIRGs), found at sub-mm wavelengths, is difficult with current telescopes, although Spitzer observations have revealed the power of the mid-infrared in ULIRG and AGN identification (e.g., Ivison et al. 2004). ULIRGs at $z>5$ will remain unidentified until JWST, even though they may well already be present in today's sub-mm samples. Midinfrared imaging will penetrate dust obscuration and test whether mergers are the cause of the energy injection in high redshift ULIRGs. Near-infrared spectroscopy with JWST will have the capability to measure redshifts for identifications that cannot be secured from ground-based spectroscopy, and will yield kinematic information on the merging system. Mid-infrared spectroscopy will be able to diagnose the energy sources in the interior of the systems, as starbursts have strong PAH features, while AGN have strong $[\mathrm{NeVI}] 7.66 \mu \mathrm{m}$ lines.

\section{The birth of stars and protoplanetary systems}

JWST seeks to unravel the birth and early evolution of stars, from infall onto dustenshrouded protostars, to the genesis of planetary systems. The formation of stars and planets is a complex process, even in the well-developed paradigm for a single, isolated low-mass star. Things are even more complicated, as stars very rarely form in isolation. The current picture of star formation starts on large scales, as molecular cloud cores cool and fragment to form highly dynamic clusters of protostars, spanning the mass spectrum from $\mathrm{O}$ stars to planetary-mass brown dwarfs. Within those clusters, individual young sources are often encircled by disks of warm gas and dust, where material aggregates to form proto-planetary systems. These disks are the source of highly-collimated jets and outflows, which transfer energy and angular momentum from the infalling material into the surrounding medium, and clear away the remainder of the birth core. On larger scales, 
the intense ultraviolet flux and strong winds of the most massive stars can disperse an entire molecular cloud, while simultaneously ionizing and evaporating the circumstellar disks of the surrounding lower-mass stars.

\subsection{How do protostellar clouds collapse?}

Stars form in small $(\sim 0.1 \mathrm{pc})$ regions undergoing gravitational collapse within larger molecular clouds. Standard theory predicts that these cores collapse from the inside out, and the slowly collapsing and rotating core approximates a singular isothermal sphere, breaking down in the center where a protostar and disk are found. Alternatives to the standard picture include ambipolar diffusion, due to incomplete coupling of magnetic fields to the gas, and pressure-confined or chaotic fractal cores. These different models predict different density distributions for star-forming cores. By measuring the extinction to background stars, JWST will map those density distributions for cores in a wide range of environments and evolutionary states, in order to understand the relative roles that magnetic fields, turbulence, and rotation play while the clouds collapse to form stars. Once self-gravitating molecular cloud cores have formed, they collapse to form protostellar seeds, which gain material via continuing accretion. These protostars will begin radiating in the $10 \mu \mathrm{m}$ to $20 \mu \mathrm{m}$ region, since radiation from the warm central source is scattered off dust grains in the inner envelope into the line-of-sight. The degree of scattering reveals the density distribution in the envelope.

\subsection{How do very high and very low-mass stars form?}

The formation of massive stars produces intense winds and ionizing radiation which impacts the surrounding molecular cloud material and the nascent circumstellar disks of adjacent low-mass stars. The mechanism by which massive stars form is not yet known. The standard disk-accretion scenario appropriate for low-mass stars cannot be simply scaled up, since predictions are that the radiation pressure from the growing central source would build up so quickly that no more material could accrete, limiting the mass of the source (Yorke \& Sonnhalter 2002). Likewise, the low-mass opacity-limited cutoff for star formation is predicted to be 3 to $10 \mathrm{M}_{\mathrm{JUP}}$, although a more detailed consideration of the role of magnetic fields may bring this down to $1 \mathrm{M}_{\text {JUP }}$. JWST will conduct imaging and spectroscopic surveys of young star-forming regions to establish both the high and low-mass limits of the initial mass function, and to determine the processes that operate at the extremes of star formation. In addition, free-floating objects less massive than 10 MJUP can serve as important proxies for true planets.

\subsection{How do protoplanetary systems form?}

Direct imaging of disks around low-mass stars reveals their internal density and temperature structure, and shows how disks are affected by their ambient environment. Since circumstellar disks are both a product and a mediator of the star formation process, as well as the progenitors of planetary systems, it is clear that a fuller understanding of the evolution of circumstellar disks will play a key role in our understanding of these central topics. Can these disks form planets before being destroyed? How are gas and dust processed through stars and returned to the interstellar medium? Spectra of circumstellar disks will trace the formation history of planetesimals and other solid bodies, their composition, their processing, and their possible future evolution. 


\section{Planetary systems and the origins of life}

JWST seeks to determine the physical and chemical properties of planetary systems including our own, and to investigate the potential for the origins of life in those systems.

\subsection{How do planets form?}

The formation of multiple objects is a common outcome of star formation, including binary or higher-order star systems, a central star orbited by brown dwarfs and/or planets, or a star with a remnant disk of particulates. Brown dwarfs and giant planets might arise from two different formation mechanisms, or planets could be the low-mass tail of a single star-formation process. The formation of giant planets is a signpost, detectable with JWST, of a process that may also generate terrestrial planets. In contrast, direct collapse formation of brown dwarfs may signal systems in which terrestrial planet formation is rare or impossible, because of the required disk mass, angular momentum and subsequent disk evolution. JWST will conduct coronagraphic surveys of nearby stars to find mature Jovian companions, and of more distant systems to find young planets. Spectroscopy of isolated or widely separated planets or sub-brown dwarfs will reveal basic physical parameters such as gravity, composition, temperature and the effect of clouds. The remnant of the circumstellar disk that formed our Solar System is observable today in the form of smaller planets, moons, asteroids and comets, along with the zodiacal light and interplanetary gas and dust. JWST spectroscopy of comets will reveal how circumstellar disks are like our own Solar System.

\subsection{How are habitable zones established?}

Some geochemical evidence suggests that Earth's water did not come from locally formed planetesimals. The source of water is uncertain; it could have been delivered through impacts of asteroids and comets, although isotopic ratios call this into question. By measuring isotopic ratios in comets and larger Kuiper Belt bodies, JWST can solve this part of the puzzle, removing a major uncertainty in the source of water for our own planet. JWST will also monitor icy bodies in the outer Solar System, such as Titan, in order to investigate chemical processes in the early Solar System.

\section{JWST implementation}

JWST will be a large cold telescope, with a wide field of view, exceptional angular resolution and sensitivity, and wide wavelength coverage in both imaging and spectroscopy. It will be launched early in the next decade to an orbit around the Earth-Sun second Lagrange point (L2). The scientific objectives can only be met with JWST; no other existing or planned ground-based telescope or space telescope mission can meet these requirements. In this section we describe the design of the JWST observatory and mission.

The JWST Project is organized into three segments: observatory, ground and launch. The observatory is composed of an optical telescope element (OTE), an integrated science instrument module (ISIM) containing the scientific instruments, a spacecraft, and a sunshield (Figure 1). The OTE is a deployable optical system that provides diffractionlimited performance at $2 \mu \mathrm{m}$ using active wavefront sensing and control (WFS\&C). The ISIM contains a near-infrared camera (NIRCam), a near-infrared multi-object spectrograph (NIRSpec), a mid-infrared camera (MIRI), and a near-infrared tunable filter imager (TFI). The ISIM also contains a fine guidance sensor (FGS) to provide active control of pointing. The spacecraft provides the pointing platform and housekeeping functions for the observatory. The wavelength range of JWST spans 0.6 to 27 microns, limited at the 


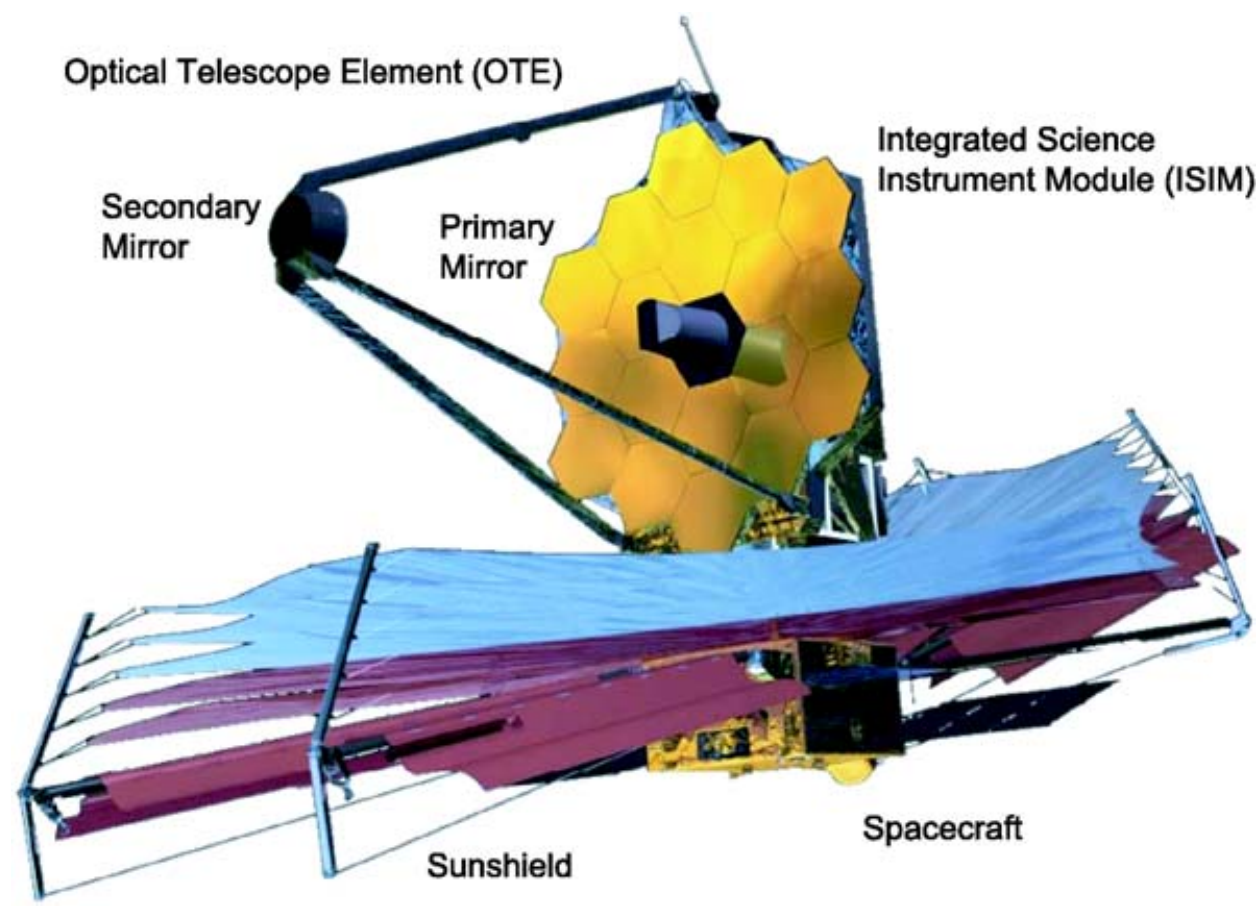

Figure 1. The JWST Observatory. The optical telescope element (OTE) contains the primary and secondary mirrors, the integrated science instrument module (ISIM) element contains the instrumentation, and the spacecraft element consists of the spacecraft and the sunshield.

short end by the gold coatings on the primary mirror and at the long end by the detector technology. The sunshield shades the OTE and ISIM from solar illumination to allow operation with high sensitivity out to $27 \mu \mathrm{m}$, and provides a stable thermal environment for the OTE and ISIM. For details on the design, see Sabelhaus \& Decker (2004) and subsequent papers in that volume.

\subsection{Observatory}

The primary mirror is made up of 18 beryllium segments (Figure 2), each positioned by actuators with 6 degrees of freedom plus radius of curvature adjustment. The $\mathrm{f} / 20$ three-mirror anastigmat telescope has a $25 \mathrm{~m}^{2}$ collecting area, and delivers a diffractionlimited Strehl ratio $>0.80$ at 2 microns and longer wavelengths. Most broad-band imaging observations at $<10 \mu \mathrm{m}$ wavelengths will have zodiacal background-limited sensitivity. The sunshield will passively cool the telescope to below $50 \mathrm{~K}$, with the NIR instruments at 37 to $40 \mathrm{~K}$. The MIRI will be actively cooled with a cryo-cooler to $7 \mathrm{~K}$. The field of regard for the telescope will enable all regions of the sky to be viewed during the year, and a continuous viewing zone within 5 degrees of each ecliptic pole will be available. JWST will be able to find a guide star $95 \%$ of the time for any available target at any available roll angle. The observational efficiency is designed such that $70 \%$ of the time the observatory will be available to make science observations, although this will depend on the particular program selected (and the consequent number of slews).

The WFS\&C subsystem aligns the mirror segments on-orbit so that their wavefronts match properly, creating a diffraction-limited 6.5-m telescope, rather than overlapping images from 18 individual $1.3 \mathrm{~m}$ telescopes (Figure 3). Determination of the wavefront error and the necessary OTE mirror commands is done on the ground using downlinked 


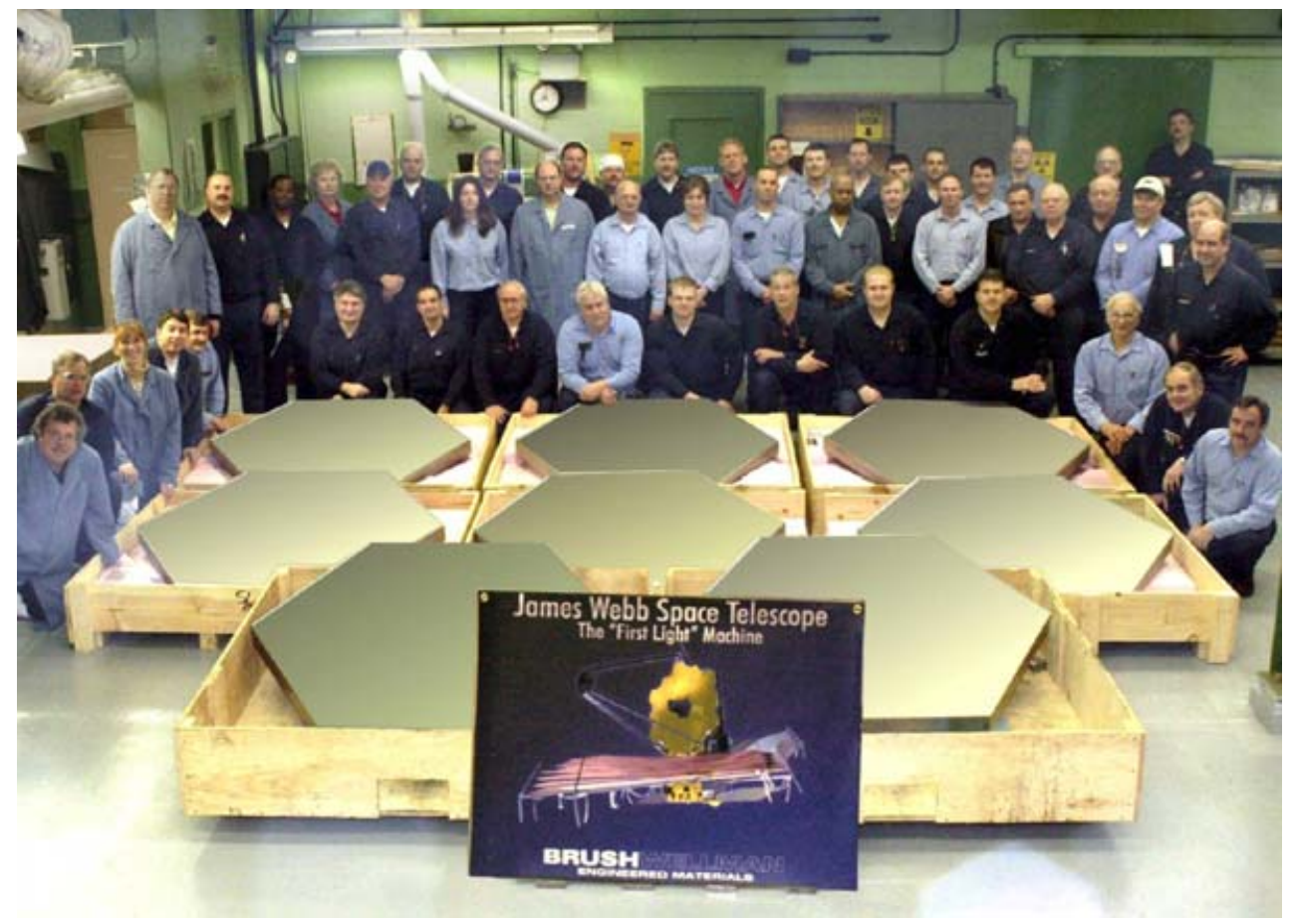

Figure 2. The JWST project has recently reached a milestone with the construction of all 18 primary mirror segments from beryllium blanks. The segments have been lightweighted, a process which removes $95 \%$ of the original material, resulting in a final density of $25 \mathrm{~kg} / \mathrm{m}^{2}$. The mirror segments are currently undergoing shaping and polishing.

image data. The algorithm uses least squares fits to images taken in and out of focus, at different wavelengths, and at multiple field points. The focus is adjusted using weak lenses in the NIRCam filter wheel. This algorithm was used to diagnose and measure the spherical aberration of the HST primary mirror.

\subsection{Instrumentation}

The integrated science instrument (ISIM) module contains the science instruments for the observatory, and the support electronics for the science instruments: NIRCam, NIRSpec, TFI, and MIRI. A cryo-cooler will be used for cooling MIRI and its Si:As detectors. The near-infrared detector arrays in the other instruments are passively cooled HgCdTe. In addition to the science instruments, the ISIM contains the fine guidance sensor (FGS) and the computer that directs the daily science observations based on plans received from the ground. Simultaneous operation of all science instruments is possible; this capability will be used for parallel calibration, including darks and possibly sky flats.

\subsubsection{Near-Infrared Camera}

NIRCam provides filter imaging in the 0.6 to $5.0 \mu \mathrm{m}$ range with high sensitivity, wavelength multiplexing, and wide field of view. It includes the ability to sense the wavefront errors of the observatory. NIRCam consists of two fully redundant, identical optical trains mounted on two beryllium benches. The incoming light passes through a dichroic, which is used to split the light into the short $(0.6$ to $2.3 \mu \mathrm{m})$ and long $(2.4$ to $5.0 \mu \mathrm{m})$ wavelength light paths. Each of these two beams then passes through a pupil wheel and filter wheel combination, before being imaged onto the detectors. The detectors are eight $2 \mathrm{~K}$ arrays 

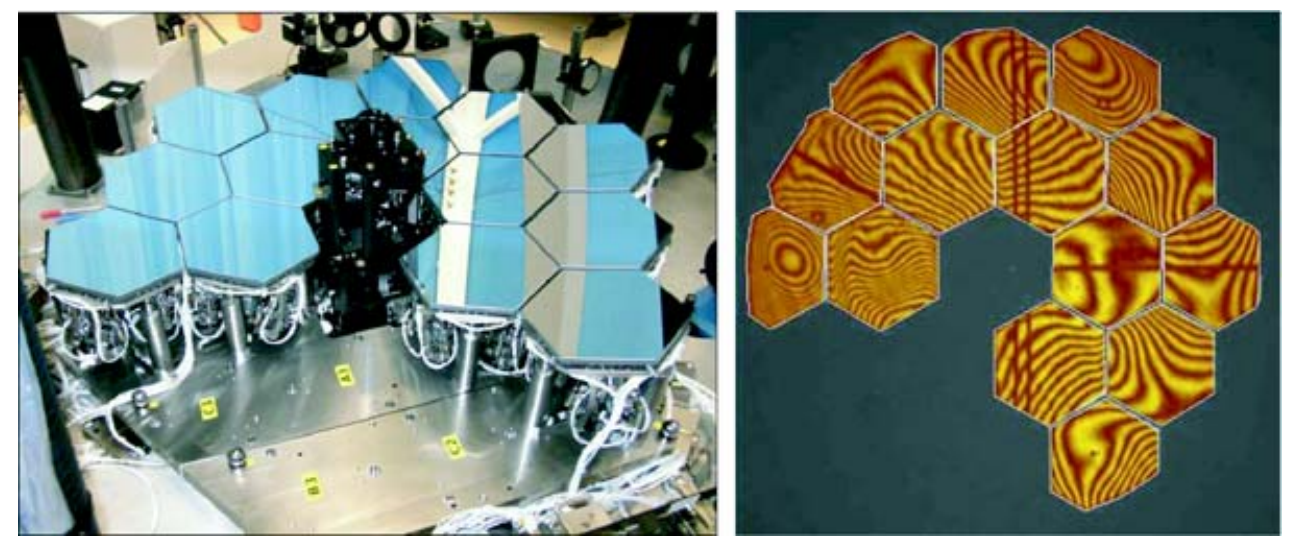

Figure 3. The JWST project uses a ground-based 1/6-scale testbed to determine the correct wavefront-sensing and control algorithms. Currently, 14 segments are installed in the testbed, which will be a scale model of the full primary mirror when completed.

sampling the PSF with 32 mas pixels in the short wavelength channel and two $2 \mathrm{~K}$ arrays with 65 mas pixels at long wavelengths, delivering a simultaneous $2.2 \times 4.4$ arcmin field of view. Coronagraphic imaging is possible using a set of spots in the focal plane and Lyot stops in the pupil wheel. Current models predict a coronagraphic contrast of $\sim 10^{4}$ at 0.5 arcsec, at a wavelength of $4.6 \mu \mathrm{m}$.

\subsubsection{Near-Infrared Spectrograph}

NIRSpec is a near-infrared multi-object dispersive spectrograph capable of simultaneously observing more than 100 sources over a field-of-view (FoV) of $3.4 \times 3.1$ arcmin. The multi-object capability is provided by a micro-shutter array of 750 (spectral) by 350 (spatial) individually addressable shutters with $200 \times 450$ milliarcsec openings and $250 \times 500$ milliarcsec pitch. In addition, NIRSpec includes fixed slits and an $3^{\prime \prime} \times 3^{\prime \prime}$ integral field unit for imaging spectroscopy. Six gratings will yield resolving powers of $\mathrm{R}$ $\sim 1000$ and $\sim 3000$, over 1.0 to $5.0 \mu \mathrm{m}$. A single prism will yield $\mathrm{R} \sim 100$ over 0.6 to $5.0 \mu \mathrm{m}$. NIRSpec uses two $2 \mathrm{~K} \mathrm{HgCdTe} \mathrm{detector} \mathrm{arrays} \mathrm{with} \mathrm{100mas} \mathrm{pixels.}$

\subsubsection{Mid-Infrared Instrument}

The Mid-Infrared Instrument (MIRI) on JWST provides imaging and spectroscopic measurements over the wavelength range 5 to $27 \mu \mathrm{m}$. The imaging module provides broadband imaging, coronagraphy, and low-resolution $(\mathrm{R} \sim 100,5-10 \mu \mathrm{m})$ slit spectroscopy using a single $1024 \times 1024$ pixel Si:As sensor chip assembly (SCA) with 110 mas pixels. The imaging region will provide a field of view of $84 \times 113$ arcsec. MIRI imaging includes three coronagraphic phase masks for a quadrant-phase coronagraph and one opaque spot for a Lyot coronagraph. The coronagraphic masks each have a square field of view of $26 \times 26$ arcsec and are optimized for particular wavelengths.

The MIRI integral field spectrograph uses dichroics and image slicers to obtain simultaneous $\mathrm{R} \sim 3000$ spectral and spatial data on a field of view that varies between 3" and 7 " on a side, depending on wavelength. A full 5 to $28 \mu \mathrm{m}$ spectrum of a single field of view is obtained by taking exposures at each of three settings of the grating wheel. MIRI spectroscopy uses two $1 \mathrm{~K}$ Si:As detector arrays. 


\subsubsection{Tunable Filter Imager}

The tunable filter imager (TFI) provides narrow-band imaging in the wavelength ranges $1.9<\lambda<4.8$ over a field of view of $2.2 \times 2.2 \operatorname{arcmin}^{2}$ with a spectral resolution $\mathrm{R} \sim 100$. The TFI team is currently studying the possibility of extending the wavelength range to shorter wavelengths. The TFI uses dielectric-coated Fabry-Perot etalon plates with a small air (vacuum) gap. The finesse is about 30 and the filters are used in third order. The TFI incorporates four coronagraphic occulting spots permanently on one side of the field of view. The coronagraph will deliver a contrast ratio of $\sim 10^{4}(10 \sigma)$ at 1 arcsec separation. The sensitivity is limited by speckle noise. Contrast ratios of $10^{5}$ may be achievable at sub-arcsec scales using roll or spectral deconvolution techniques.

\subsubsection{Fine Guidance Sensor}

The fine guidance sensor (FGS) instrument uses two fields of view in the JWST focal plane to provide fine guidance for the telescope. Roll is sensed by star trackers on the spacecraft bus. The optical assembly of the FGS instrument consists of two channels, imaging separate regions of the sky onto independent $2 \mathrm{~K} \mathrm{HgCdTe}$ detectors with 67 mas pixels. The field of view is $2.3 \times 2.3 \mathrm{arcmin}^{2}$. JWST will be capable of relative pointing offsets with an accuracy of 5mas rms, which will enable sub-pixel dithering and coronagraphic acquisition. Absolute astrometric accuracy will be limited to 1 arcsec rms by the accuracy of the guide star catalog.

\subsection{Launch, Commissioning and Operations}

JWST will be launched on an Arianespace Ariane 5 ECA rocket into orbit about the second Lagrange (L2) point, approximately $1.5 \times 10^{6} \mathrm{~km}$ from the Earth in the EarthSun system. JWST will have a 6 month orbit period about the L2 point in the rotating coordinate system moving with the Earth around the Sun. Station-keeping maneuvers are performed after the end of each orbit determination period of 22 days. The total observatory mass is $6500 \mathrm{~kg}$, including station-keeping propellant.

The observatory has the following five deployments: (1) deploy spacecraft bus appendages, (2) deploy sunshield, (3) extend telescope tower, (4) deploy secondary mirror support structure, and (5) deploy primary mirror wings. The deployment mechanism design includes heaters and other protections that eliminate the need for time critical events and allow for unlatching and re-latching to relieve any residual long-term stress in the structure. Following deployment, the mirrors will be aligned through wavefront sensing and control. Commissioning will be complete 180 days after launch. JWST will operate with all science instruments for at least five years after completion of commissioning, and to maintain the possibility of a longer mission lifetime, will carry propellant sized for at least 10 years of operation after launch. There are no other consumables which would limit lifetime.

JWST will be operated from a Science and Operations Center (S\&OC) located at the Space Telescope Science Institute, the organization that operates HST for NASA. Although the capabilities of JWST are being developed to address science themes discussed in the previous sections, about $85 \%$ of the observing time on JWST will be awarded through a series of proposal solicitations, which will be open to any astronomer in the world. The scope of JWST's competitively-selected investigations will range from large legacy-style projects, which last months and address a range of science goals simultaneously, to small programs that target important but very specific science objectives. The observatory will be operated through an event-driven architecture, with very little real-time commanding. Observation plan uplinks will be weekly, while data downlinks 


\section{Comparative performance of JWST with a 30m GSMT and Spitzer}

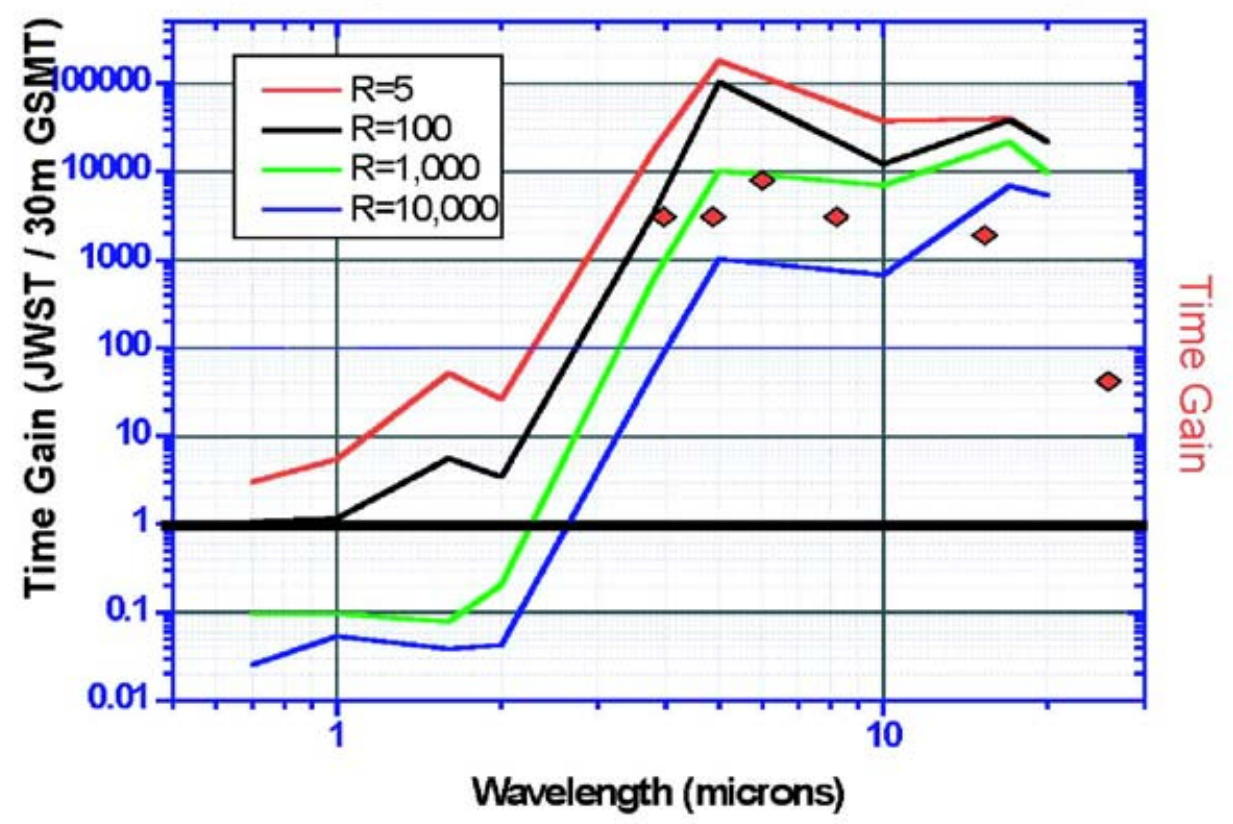

Figure 4. A comparison of the performance expected for a 30m ELT with that of JWST and Spitzer. JWST is more sensitive for broad-band imaging in the near-infrared, and all observations in the thermal mid-infrared. For high spectral resolutions and observations in the optical, an ELT is more powerful. JWST is designed to make observations that are not feasible from the ground, and will conduct a science program that is complementary to that of an ELT.

will be daily, using the Deep Space Network. The STScI will pipeline calibrate the data and distribute them to the astronomers through the World Wide Web.

\section{Synergy with ELTs}

JWST is designed to have complementary capabilities to those of an Extremely Large Telescope (ELT) on the ground. JWST advantages include very high sensitivity imaging, continuous wavelength coverage from 0.6 to 27 microns, full-sky coverage with high observing efficiency, a stable diffraction-limited point spread function and high dynamic range. In comparison to JWST, the advantages of a 30m ELT include blue and optical sensitivity, 25 times the collecting area and up to 5 times the spatial resolution, very high spectral resolutions of 100,000 or more, higher sensitivity at R > 1000 in the near infrared, and the ability to upgrade the instruments over time. Figure 4 shows a comparison of the sensitivity expected for JWST and a 30m ELT on the ground. 


\section{References}

Cen, R. 2003, ApJ 591, L5

Fan, X., et al. 2002, AJ 123, 1247

Ferrarese, L. \& Merritt, D. 2000, ApJ 539, L9

Ivison, R.J., et al. 2004, ApJS 154, 124

Kogut, A., et al. 2003, ApJS 148, 161

Rees, M.J. 1997, in: N. R. Tanvir, A. Aragon-Salamanca \& J. V. Wall (eds.), The Hubble Space Telescope and the High Redshift Universe, (World Scientific: Singapore), 115

Sabelhaus, P.A. \& Decker, J. 2004, in: J. C. Mather (ed.), Optical, Infrared, and Millimeter Space Telescopes, Proceedings of SPIE, 5487, (SPIE: Bellingham WA), 550

Shu, F.H., Adams, F. C. \& Lizano, S. 1987, ARA\& A 25, 23

Yorke, H.W. \& Sonnhalter, C. 2002, ApJ 569, 846

\section{Discussion}

BERGeron: You mentioned very deep, small field surveys, but JWST is also suited to do a larger area $\left(\sim 1 \mathrm{deg}^{2}\right)$ deep survey aimed at detecting high redshift $(z>7)$ galaxies. Are there any plans to do such a survey?

GARDNER: The observing program for JWST will be selected through peer-reviewed proposals, in a manner very similar to HST. We expect there will be some type of Legacy or Treasury or other very large proposal opportunity. The field of view of NIRCam is comparable to that of the Advanced Camera for Surveys on HST, and just as the COSMOS survey on HST covered 2 square degrees, a similar survey with JWST will be possible, if approved by the time allocation committee.

SCHMIDT: When comparing ELT (or TMT) and JWST sensitivities, what assumptions were made on the ELT side, with regard to Strehls? Were they optimistic or conservative?

GARDNER: We attempted to be realistic. We assumed MCAO, but did not assume $\mathrm{OH}$ supression in imaging.

HARTUnG: Concerning the Strehl ratio of 0.8 in K: does this number mainly come from the fact of having the segmented mirrors, or is the fact of having segmented mirrors already included in the theoretical Strehl ratio (i.e., $\mathrm{SR}=100 \%$ with segments in the ideal case) and the $20 \%$ of loss is mainly due to the residual errors after putting the mirrors into phase?

GARDNER: The Strehl ratio of 0.8 is relative to a perfect segmented mirror, and the 0.2 comes from residual wavefront error.

GRIFFITHS: What factors have resulted in the increased cost projections?

GARDNER: The current cost estimate for JWST is $\$ 4.5 \mathrm{~B}$, including all of the design work already done and 10 years of operations. This represents an increase in the estimate of about $\$ 1 \mathrm{~B}$ since 2003 , which includes $\$ 180 \mathrm{M}$ for a re-evaluation of the integration and test plan, $\$ 95 \mathrm{M}$ for instruments, $\$ 210$ of increased contingency (now totaling $23 \%$ ), and $\$ 520 \mathrm{M}$ for a 22 month launch slip to June 2013. The launch slip is due to a delay in the US government approval to launch JWST on an ESA-provided Ariane 5, budget profile limitations in 2006 and 2007, and schedule risk reduction. The JWST project examined the possibility of descopes to the mirror size, but since the mirror segments are already 
made, this would not save much money. In order to reduce risk, JWST's technology development efforts remain on the previous schedule, and were not slipped by the 22 months.

ZINNECKER: Is there a journal publication of the science case?

GARDNER: Yes, a publication is currently in the refereeing process, and will be posted to astro-ph upon acceptance.

ZINNECKER: What is the sensitivity of JWST, e.g., in the thermal infrared? How far away in the universe can you see a protostar?

GARDNER: Imaging sensitivities are: $10.4 \mathrm{nJy}$ at 2 microns, $0.7 \mu \mathrm{Jy}$ at 10 microns and $7.3 \mu \mathrm{Jy}$ at 21 microns. Spectroscopic sensitivities are $1.6 \times 10^{-18} \mathrm{erg} \mathrm{s}^{-1} \mathrm{~cm}^{-2}$ for $\mathrm{R}=1000$ at 2 microns, and $1.0 \times 10^{-17} \mathrm{erg} \mathrm{s}^{-1} \mathrm{~cm}^{-2}$ for $\mathrm{R}=2400$ at 9.2 microns. These are all $10 \sigma$ in 10000 seconds.

IYE: You have many steps to deploy sun-shades and segment assembly. Does each step have a backup system in case of failure? What is the risk management? Is there something special?

GARDNER: All of the deployments are done while the observatory is still warm, and there are heaters to ensure this. The deployments can be done in both directions until the final latching, and all of the motor windings are redundant. The observatory will be thoroughly tested on the ground. All interfaces and requirements are under configuration control, and are tracked through NASA's system engineering procedures. 